\title{
Early care and education in disadvantaged contexts (birth to 9)
}

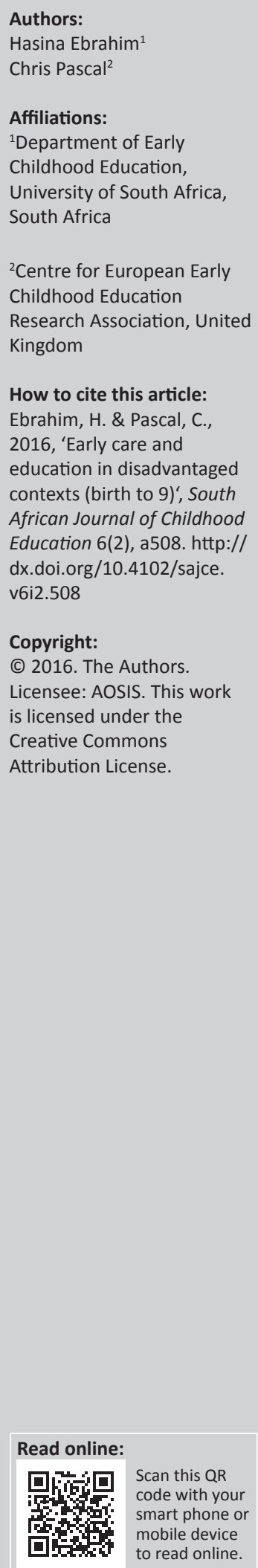

This special issue on early childhood care and education (birth to 9) in disadvantaged contexts is timely and critical, taking into account the urgency to deal with the constellation of risks facing young children and their families in vulnerable circumstances. Interventions directed at poor children and their families continue to be challenged by complexities, noted as identifying relevant conceptualisations for action, being responsive to socio-cultural diversity and dealing with socio-economic circumstances that stratify societies. In this issue, 10 articles from different countries, in their varying emphasis on early care and education in disadvantaged contexts, seek to highlight critical issues that need to be considered for more equitable opportunities and outcomes through the vantage point of the early years. The discussion that follows is arranged in three themes, namely highlighting dominant framings/responses, suggestions for contextual intervention and methodological issues in researching the vulnerable.

A unifying thread in all articles is the examination of dominant framings and its limitations for thought and actions for children in disadvantaged contexts. Muthukrishna and Naidu in arguing for a social sustainability lens contend that narrow conceptions of child well-being have skewed the focus of interventions for dealing with poor children and their families. Also, on the issue of conceptions, Nyamukapa argues that narrow conceptions of child poverty in sub-Saharan Africa do not adequately take into account the household dynamics and in-house distributions when targeting young children in social protection. Okwany, Munene and Okwany as well as Wadende all argue for a critical stance to dominant western narratives of early care and education which sidelines local ways of knowing and being that are important for developing contextualised policy and culturally responsive practice in Kenya and Uganda. Keang and Vong highlight how the kindergarten education system in a rural community in China marginalises history and culture as resources for developing appropriate approaches to early education.

The focus on teachers, their empowerment and instructional practices also lays bare the need to disable dominant framings or responses to address the local realities of educational achievement of children in disadvantaged contexts. Author groups focus on the problematics in early language and literacy development. Mzimela and Ramrathan make explicit how the rural context defies the use of single-grade arrangements for instruction in early reading as they examine the phenomena of multi-grade classrooms. Grossen, Muthukrishna and Naidoo show contestations that arise in choosing an effective instructional model for addressing English proficiency of Samoan children. Martin and Ebrahim argue that the dominant discourse on literacy as an autonomous skill interwoven with the image of children as adults-in-the-making and the maturationistenvironmental view of readiness discounts literacy as social practice. Bose and Seetso shift the focus from literacy to the development of resource materials for preschool teachers in Maths and Science. The authors argue that resources for practice would be impoverished if they did not take into account teachers' content and pedagogic knowledge of subjects.

In the quest to decentralise dominant framings or responses, the articles in this issue focus on the gaps for more responsive interventions. In so doing, they bring marginalised perspectives to the fore. Several authors argue for a focus on contextual responsiveness to strengthen early care and education policy and practice. Okwany provides evidence of how indigenous resources and informal safety net systems in communities can be accessed to intervene in the lives of the poor. In describing the Kenyan experience, Munene and Okwany suggest that the divide between health and education is best addressed through engaging with assumptions that lead to silo mentality in service provision as well as privileging local knowledge and practices as community assets for building responsive policies and practices. Naidoo and Muthukrishna in arguing for a social sustainability lens show the importance of approaching child well-being interventions from value-driven premises which takes into account systemic, contextual and community assets. In keeping with the sustainability argument, Wadende highlights the importance of using indigenous motivational care-giving practices embedded in an unwritten curriculum and community 
participation in early childhood centres to assist young children in home-school transitions. Keang and Vong note that the formalisation of kindergarten education in the rural villages of China would best be effected through paying careful attention to multiple factors affecting quality provision for vulnerable communities. Nyamukapa draws attention to the need for targeted social protection for young children in Zimbabwe, taking into account the dependency load and size of households as they impact on what becomes available to children amongst other efforts to building a comprehensive system for early childhood development.

This special issue also shows that the way forward lies in focusing on teachers and their instructional practices and abilities to deal with contextual realities to forge navigational maps to improve the lives of children in vulnerable circumstances. Mzimela and Ramrathan illuminate the practice of reading in multi-grade classes as a feature of rural schools and call for a focus on teacher agency and specifically their decision-making abilities to guide developmental needs. Bose and Seetso stress the need for early childhood teachers to be involved in their own development so that support material can be developed from their starting points, keeping in mind the needs of children in their cultural context. Through a quantitative study which compared effects of textbased with standards-based instruction of English, Grossen, Muthukrishna and Naidoo found that with Somoan children who had no English competence, it was best to address their needs through text-based instruction which provides structure, clear expectations, focused tasks and direct explicit instruction where no assumptions are made about prior knowledge of English. In summary, the call for teacher education to be cast within a social justice framework, as noted by Martin and Ebrahim, echoes the importance of keeping equality in opportunities and outcomes high on the reform agenda for early care and education.

The articles in this issue also highlight methodological issues in knowledge production about early care and education in disadvantaged contexts. Ten articles adopt a qualitative approach to engage with the subjective perspectives of research subjects. One article uses the quantitative approach which is valuable in comparing models of intervention and determining effectiveness. The qualitative approach is also further qualified by highlighting specific elements used to develop a more bottom-up inquiry. Muthukrishna and Naidoo advocate for a participatory research approach which addresses power imbalances between researchers and participants that arise in marginalised communities. In the same vein, Okwany makes a case for appreciative enquiry to co-construct and generate ideas for change in communities. The articles show that a variety of techniques such as semi-structured interviews, indepth interviews, questionnaires, focus group interviews, observations, field notes, narratives, social mapping, ranking excercises, body mapping, photo voice and visual data are helpful in producing data. Working with subjects in marginalised communities requires different lines of action. For example, in studies by Muthukrishna and Naidoo as well as by Keang and Vong, the use of indigenous languages was important to make participants' views and experiences accessible. Highlighting and interrogating the ethics of dealing with children and adult vulnerabilities, both in research and practice, is also important if we are to truly develop more participatory and rights-based approaches to knowledge creation and praxis in early childhood education and care.

This special issue amplifies the need to continue knowledge production about early care and education in disadvantaged context, especially from the Global South. This task is important bearing in mind the complexities in the care and education environment together with rapid changes in societies which makes it difficult to adopt a single approach to children, their families and early childhood services. The main narrative emerging from the articles is the need for policies, services and practices to be contextually responsive but professionally and scientifically informed. The values, beliefs and educational practices embedded in families and communities are important to connect with in order to address unmet needs. As children make transitions to early childhood centres and schools, it is imperative to pay attention to the infrastructure available, the competence of teachers as secondary socialisation agents and the models used to educate young children growing up in vulnerable circumstances. 